\title{
THE UTR-2 VERY LOW FREQUENCY SKY SURVEY AND ITS MAIN RESULTS
}

\author{
K.P. SOKOLOV \\ Institute of Radio Astronomy, \\ Ukrainian National Academy of Sciences
}

\section{Introduction}

During the past decade there has been a dramatic increase in the amount of high-frequency $(\nu>1000 \mathrm{MHz})$ data currently underlying the studies of bright compact sources $\left(T_{b} \sim 10^{11-12} K, l \leq 1 \mathrm{kpc}\right)$ with flat spectra. But in order to determine physical conditions inside extragalactic radio sources at different stages of their evolution the studies of old extended $(l \sim 100 \mathrm{kpc})$ sources with low surface brightness and steep spectra which constitute the dominant radio source population at very low frequencies $(\nu \ll 100 \mathrm{MHz})$ are also needed. These sources are known to represent the final stage in the evolution of extragalactic objects.

On the other hand, from the literature data it follows that extended sources observed at very low frequencies form one of the most astrophysically significant class of objects for studies of the general properties of extragalactic radio source space distribution, because: (a) these sources constitute the so-called "parent population" of extragalactic objects whose observed parameters are not biased by relativistic effects; (b) these sources are characterized by the least scatter in their luminosities (Chambers et al., 1988); (c) these sources have a lifetime $t \sim 3 \cdot 10^{9}$ years (Cordey, 1986) which is comparable with the period passed from the suggested epoch of radio source initial formation in early Universe.

Nevertheless these radio sources represent one of the least studied classes of extragalactic objects. The main goal of the UTR-2 Very Low Frequency Sky Survey is to provide the database on radio sources radiating at very low frequencies for further studies of their physics and statistical properties. 


\section{Discussion}

Since 1973 the very low-frequency radio telescope UTR-2 has been used for radio sky surveying at six frequencies within the range 10 to $25 \mathrm{MHz}$. The UTR-2 Sky Survey has covered now two regions of the sky within the declination zones $-13^{\circ}$ to $20^{\circ}$ and $40^{\circ}$ to $60^{\circ}$ (Braude et al., 1994). The total solid angel of these regions is about $4 \mathrm{sr}$. The maximum sensitivity of the survey is $20 \mathrm{Jy}$ at $25 \mathrm{MHz}$.

The radio source catalogue of the UTR-2 Sky Survey contains estimates of coordinates and flux densities of more than 2000 sources at 10, 12.6, $14.7,16.7,20$ and $25 \mathrm{MHz}$. About 20 per cent of the sources observed can not be identified with objects from other higher-frequency surveys. The highly reliable and statistically complete samples of extended extragalactic radio sources radiating at very low frequencies have been selected on the basis of the data obtained. It should be noted that sources from the UTR-2 samples are weaker than those from the well-studied 3CR-sample of strong sources. The data obtained have been used to study space distribution of extended extragalactic radio sources and their angular structures.

On the basis of the UTR-2 data the statistical relationship $n(S)$ or source counts at $25 \mathrm{MHz}$ have been obtained and physical reasons responsible for the strong cosmological evolution effects seen at very low frequencies have been considered (Sokolov 1988). The analysis of the $25 \mathrm{-MHz}$ source count has confirmed the suggestion mentioned in the literature many times that effects of radio source cosmological evolution should manifest themselves particular strongly at very low frequencies. It has been shown that the $25-\mathrm{MHz}$ source count for strong radio sources is conditioned mainly by the nearby powerful radio galaxies. On the contrary, strong evolution effects seen for weak radio sources are conditioned by quasars and unidentified objects. This result differs in principal from those observed at high frequencies where the relative content of radio galaxies and quasars remains practically constant within the wide flux density intervals where the corresponding source counts are analyzed. The analysis of the $25-\mathrm{MHz}$ source count has also allowed us to suggest the existence of a decrease in the space density of the most distant extended radio sources. In view of similarity in the general pattern of cosmological evolution of radio sources of different morphological types the result obtained for extended sources found at very low frequencies indicates on the existence of a redshift cut-off in the space distribution of the whole class of extragalactic sources.

For further implication of the results obtained the study of the astrophysical significance of the source counts observed in different frequency ranges has been carried out (Sokolov,1990). It has been shown that general properties of radio source spatial distribution manifest themselves most dis- 
tinctly at very low frequencies since the physical conditions for the existence of a "flux density - distance" correlation, which constitutes a basis for every source count analysis, are best satisfied for extended isotropically radiating objects. On the contrary, this correlation cannot exist in principal for bright compact sources, since their observed flux densities are conditioned mainly by Doppler enhancement rather than distance. In the framework of the study explanations were make of: (a) the frequency dependence of the overall shape of the source counts, (b) the cosmological paradox caused by the qualitatively different character of the observed spatial distribution for compact and extended radio sources, (c) the difficulties of detecting the reality of a redshift cutoff from analysis of high-frequency source counts.

Further qualitative studies of the space distribution of extended sources suggest evaluation of the RLF for weak sources which constitute the maximum region in the $25-\mathrm{MHz}$ source count. To reach this aim and to study the physical nature of unidentified radio sources with very steep spectra the complete sample of 265 weak $\left(S_{16.7}>29 \mathrm{Jy}\right)$ extragalactic sources with very steep spectra $\left(\alpha_{178}^{16.7} \geq 1.0\right)$ have been observed with the VLA at 333 and $1435 \mathrm{MHz}$ in C-configuration. Detailed discussion of astrophysical aims of angular structure studies of radio sources found at very low frequencies is given in the paper by Sokolov (1993). Analysis of the VLA images obtained is in progress now. The VLA data allow us to obtain accurate positions of unidentified sources from the sample for further clarification of their physical nature and to carry out a search for: (a) very old or the so-called "fossil" radio sources which represent the final stage in the evolution of extragalactic radio sources; (b) very distant objects, as well as to study interaction of the source extended components with gaseous environment at different $z$.

\section{References}

Braude S. Ya., Sokolov K.P. and Zakharenko S.M. (1994) Decametric Survey of Discrete Sources in the Northern Sky. XI, Astrophys.Sp.Sc.213, 1.

Chambers K.C., Miley G.K. and Breugel W.J.M. (1988) 4C40.36: a radio galaxy at a redshift 2.3, Astrophys.J. 327, L47.

Cordey R.A. (1986) Radio sources in giant E and S0 galaxies Mon.Not.R.astro.Soc. 219,575 .

Sokolov K.P. (1988) Determination of the Space Distribution Parameters for Extragalactic Radio Sources observed in Decametric-Wavelength Range, Astron.J.(Russian), 63,236 .

Sokolov K.P. (1990) Analysis of the Astrophysical Significance of Radio Source Counts Obtained in Different Frequency Ranges, Australian J. Physics, 43,263.

Sokolov K.P. (1993) On Angular Structure Studies of Very Steep Spectrum Sources Found at Decametric Wavelengths, Sub-Arcsecond Radio Astronomy, Cambridge University Press, pp. 282-283 